\title{
THE RELATIVISTIC ASTROPHYSICS EXPLORER: A NEW MISSION FOR X-RAY TIMING
}

\author{
P. Kaaret(1), J. Grindlay(1), F.K. Lamb(2), E.H. Morgan(3), J.H. Swank(4), \\ W. Zhang ${ }^{(4)}$
}

1) Harvard-Smithsonian Center for Astrophysics

2) University of Illinois

3) Massachusetts Institute of Technology

4) NASA/Goddard Space Flight Center

\begin{abstract}
The great success of the Rossi X-Ray Timing Explorer (RXTE) has given us a new probe to study strong gravitational fields and to measure the physical properties of black holes and neutron stars. Here, we describe a "next-generation" x-ray timing mission, the Relativistic Astrophysics Explorer (RAE), designed to fit within the envelope of a "medium-sized" mission. The main instruments will be a narrow-field x-ray detector array with an area of at least $60,000 \mathrm{~cm}^{2}$ equal to ten times that of RXTE, and a wide-field x-ray monitor with good sensitivity and few arcminute position resolution. We describe the design of the instruments and the science which will be possible with a factor of ten increase in collecting area.
\end{abstract}

KEYWORDS: instrumentation: detectors, X-rays: general

\section{SCIENTIFIC MOTIVATION FOR A NEW X-RAY TIMING MISSION}

The Rossi X-Ray Timing Explorer (RXTE) has made substantial and unique contributions to the study of the behavior of matter in strong gravitational fields near accreting compact objects, the formation of relativistic jets, the emission mechanisms of active galactic nuclei, the evolution of neutron stars in binaries, the x-ray emission regions in cataclysmic variables, and many other aspects of high-energy astrophysics (for a review see Bradt 1999). The key feature of RXTE is a large effective area x-ray detector coupled with a high telemetry bandwidth. The prowess of RXTE for fast timing opened a new "discovery space" in rapid x-ray variability, allowing timing studies at the dynamical time scales of the innermost orbits around stellar mass compact objects, and leading to the discovery of millisecond quasiperiodic oscillations from accreting neutron stars and black holes. The large x-ray detector area also made possible many other advances such as the discovery of coherent millisecond pulsations from an accreting neutron star, and the study of rapid spectral variations, such as the $\sim 200$ s cycles in the microquasar GRS 1915+105 related to ejection of the inner regions of the accretion disk. 
The great success of the Rossi X-Ray Timing Explorer (RXTE) is a strong indication that further progress in x-ray timing will lead to new scientific advances. Here, we describe a next generation x-ray timing mission which would offer an order of magnitude increase in x-ray timing capabilities via an x-ray detector with a geometric area of at least $60,000 \mathrm{~cm}^{2}$, equal to ten times that of RXTE. The most important advances made with this order of magnitude increase in collecting area are likely to be true discoveries and thus cannot be anticipated. However, an order of magnitude increase in area would benefit many scientific investigations. Here, we describe three particular examples.

Fast quasiperiodic oscillations from black hole candidates (BHCs) have been discovered in three systems with frequencies of $67-300 \mathrm{~Hz}$ (Remillard et al. 1999). The fast QPOs from BHCs are rather weak (rms amplitudes near 1\%) and difficult to study in detail with RXTE. A number of models of the QPOs have been proposed, all of which involve strong-field general relativistic effects, but distinguishing amongst the various models will be difficult with the RXTE data. The increase in the photon statistics with RAE would make possible much more accurate measurements of the QPO parameters and their variations with time or correlations with spectral or other timing parameters. This may lead to a unique identification of the QPO generation mechanism. Understanding these QPOs would provide a unique probe of strong-field gravity.

Millisecond oscillations in x-ray bursts have been discovered from a number of neutron stars. The oscillations have periods in the range 1.7-3 ms and are interpreted as due to inhomogeneous nuclear burning of matter initially located on the neutron star surface. The burst oscillations provide a means to constrain the neutron star mass-radius relation. Currently, the best constraint comes from a deep modulation $(75 \% \pm 17 \%)$ seen in the initial $62.5 \mathrm{~ms}$ of one burst (Strohmayer et al. 1998). RAE would detect roughly 1000 counts in each oscillation cycle near the peak of a typical bright burst. This would permit detailed examination of individual oscillation cycles and allow accurate measurement of the modulation amplitude in the first few oscillation cycles. Both our understanding of the burst oscillations and constraints on the neutron star mass-radius relation would improve.

Eclipse mapping of the accreting magnetic white dwarf XY Arietis showed that the $\mathrm{x}$-ray flux emerges from eclipse egress in $<2 \mathrm{~s}$ (Hellier 1997). For the previous 15 years, the fraction, $f$, of the white dwarf surface involved in x-ray emission had been debated with values ranging from 0.001 to 0.3 . Hellier's result, obtained by combining 20 RXTE observations, shows that $f<0.002$. Using RAE, an accurate estimate could be made of the emitting region location on each egress which would allow direct mapping of movement of the emitting region. Similar mapping can also be done in neutron star and black hole binaries. The best constraints currently available on the size of the x-ray emitting regions in black hole systems come from x-ray dips (e.g. Tomsick et al. 1997). RAE would lead to significant advances in mapping x-ray emission from many different x-ray sources. 


\section{MISSION OVERVIEW}

The Relativistic Astrophysics Explorer (RAE) will consist of two scientific instruments: a large area x-ray detector and a wide-field x-ray monitor. RAE will be designed to have telemetry sufficient to transmit the large event rate and flexible operations with multiple repointings each day to permit study of transient sources and rare states of known sources.

The goal for the large area $\mathrm{x}$-ray detector is to provide an order of magnitude increase in x-ray timing capabilities relative to RXTE. The design goals are: a useful detector area of at least $6 \mathrm{~m}^{2}$, sensitivity from $2 \mathrm{keV}$ to $30 \mathrm{keV}$, absolute timing better than $10 \mu \mathrm{s}$, minimal dead time effects for sources 10 times as bright as the Crab nebula, an energy resolution of $1.2 \mathrm{keV}$ (preferably $300 \mathrm{eV}$ ) at $6 \mathrm{keV}$, no imaging, and a field of view of $1^{\circ}$ or smaller.

All-sky x-ray monitoring is needed for several reasons. First, the x-ray monitor provides continual long-term light curves. As many x-ray sources are highly variable, knowledge of the long term behavior in important in understanding the physical nature of the sources and in placing pointed observations in the context of the source state. Second, an x-ray monitor provides a means to trigger pointed observations when a selected source reaches a state of particular interest. Finally, an x-ray monitor allows discovery of new sources or new, unpredicted, outbursts of known sources. Many of the sources of interest are transients with unknown or irregular recurrence intervals. An x-ray monitor is essential to detect transient events. The design goal for the x-ray monitor is a sensitivity of several mCrab for daily observations, sufficient to monitor a large sample of AGNs $(\sim 40)$ on a daily basis.

\section{LARGE AREA X-RAY DETECTOR ARRAY}

An effective area of $6 \mathrm{~m}^{2}$ will require a total geometric detector area near $10 \mathrm{~m}^{2}$. A detector with a cross sectional area of $10 \mathrm{~m}^{2}$ and a thickness of $0.75 \mathrm{~m}^{2}$ fits within the $3 \mathrm{~m}$ diameter fairing of a two-stage Delta II. Thus, a $6 \mathrm{~m}^{2}$ detector can be accommodated in a "medium-sized" mission without a deployment mechanism.

The x-ray detector must have low mass per unit effective area, reasonable cost, highly reliable and stable operation, efficient rejection of particle backgrounds, and good energy resolution. After extensive review of the available detector technologies, we have selected silicon detectors as the most promising candidate for large format x-ray astronomy detectors. A $2 \mathrm{~mm}$ thick silicon detector provides $40 \%$ efficiency up to $30 \mathrm{keV}$ at a mass of $0.5 \mathrm{gm} / \mathrm{cm}^{2}$; this compares favorably to PCA on XTE at a mass of $90 \mathrm{gm} / \mathrm{cm}^{2}$. Silicon is widely used and can be obtained at low cost due to large economies of scale; $10 \mathrm{~m}^{2}$ of commercially available silicon strip detectors (see below) can be procured for less than US $\$ 4 \mathrm{M}$. Silicon has a low ionization potential which leads to good energy resolution and allows silicon detectors to be operated without internal amplification. While the lack of internal amplification mandates the use of low capacitance detectors and low noise electronics for good performance, it also eliminates the need for high voltage and facilitates reliable and stable operation. 
Silicon detectors can be configured in different geometries including PIN diodes, silicon strip detectors, and silicon drift chambers. Silicon strip detectors (SSDs) are widely used in particle physics. SSDs offer one-dimensional imaging which may provide a means of effective discrimination against particle backgrounds. However, SSDs employ charge collection strips which run the length of a wafer and, thus, have relatively high capacitance which leads to high electronic noise and poor energy resolution. Given mission constraints, we estimate that the best energy resolution achievable with SSDs will be $1-2 \mathrm{keV}$ at $6 \mathrm{keV}$ (see Costa et al. these proceedings).

Silicon drift chambers (SDCs) (Gatti \& Rehak 1984) have an internal electric field arranged so that electrons, produced by interaction of radiation within the silicon, drift toward a single charge collection point. SDCs have a great advantage in that the readout electrode can be made very small even if the detection area is large; thus, the readout capacitance is small, which leads to low readout noise and good energy resolution. Cylindrical SDCs, in which a single detector electrode is used to collect charge from a cylindrical drift region, are particularly well-optimized for xray spectroscopy (Rehak et al. 1985; Lechner et al. 1996). Excellent performance has been demonstrated from cylindrical SDCs in the laboratory (Gauthier et al. 1994; Fiorini et al. 1997) and in the field (Longoni et al. 1998). Operating at $-15^{\circ} \mathrm{C}$, a resolution of $155 \mathrm{eV}(\mathrm{FWHM})$ at the Mn- $K \alpha$ line has been obtained (Fiorini et al. 1997). The main question for x-ray astronomy is whether effective particle background rejection can be achieved. We currently are engaged in a technology development program to study application of SDCs to x-ray astronomy and to develop effective techniques for charged particle background rejection.

\section{CONCLUSION}

We have presented a conceptual design for a next generation x-ray timing mission and identified key technologies which must be developed. The outstanding results from the Rossi X-Ray Timing Explorer are strong motivation for a new mission with an order of magnitude increase in x-ray timing capabilities.

\section{REFERENCES}

Bradt, H.V. 1999, astro-ph/9901174

Fiorini, C. et al. 1997, Rev. Sci. Instrum. 68, 2461

Gatti, E. \& Rehak, P. 1984, NIM, A255, 608

Gauthier, C. et al. 1994, NIM, A349, 258

Hellier, C. 1997, MNRAS, 291, 71

Lechner et al. 1996, NIM, A377, 346

Longoni, A. et al. 1998, NIM, A409, 407

Rehak, P. et al. 1996, NIM, A235, 224

Remillard et al. 1999, ApJ, 517, L127

Strohmayer, T.E. et al. 1998, ApJ, 498, L135

Tomsick, J.A., Lapshov, I., \& Kaaret, P. 1998, ApJ, 494, 747 